\title{
Über stetige Functionen, die innerhalb jedes Intervalls extreme Werte besitzen.
}

Von Julius König in Budapest.

1. Es sei $f(x)$ eine in dem überhaupt betrachteten Bereiche stetige Function der reellen Variabeln $x$, von der Beschaffenheit, dass in beliebiger Nähe der beliebigen Stelle $x_{0}$ Stellen $\xi, x^{\prime}$ und $x^{\prime \prime}$ existieren $\left(x^{\prime}<\xi<x^{\prime \prime}\right)$, für welche $f\left(x^{\prime}\right)$ und $f\left(x^{\prime \prime}\right)$ zugleich größer oder kleiner als $f(\xi)$ seien.

Wegen der Stetigkeit unserer Function nimmt diese zwischen $x^{\prime}$ und $\xi$, beziehungsweise zwischen $\xi$ und $x^{\prime \prime}$ jeden Wert an, welcher zwischen $f\left(x^{\prime}\right)$ und $f(\xi)$, bezw. zwischen $f(\xi)$ und $f\left(x^{\prime \prime}\right)$ liegt. Eines dieser Intervalle $\left[f\left(x^{\prime}\right) \ldots f(\xi), f\left(x^{\prime \prime}\right) \ldots f(\xi)\right]$ enthält jedenfalls das andere, und die Function nimmt daher jeden. in diesem zweiten Intervalle enthaltenen Wert, höchstens mit Ausnahme von $f(\xi)$, sowohl zwischen $x^{\prime}$ und $\xi$, wie zwischen $\xi$ und $x^{\prime \prime}$ an, d. h. in beliebiger Nähe einer beliebigen Stelle, oder also auch in jedem noch so kleinen Intervalle, gibt es verschiedene Stellen, denen gleiche Functionalwerte entsprechen.

Man pflegt diese Eigenschaft der Function $f(x)$ auch anders auszudrücken. Gehen wir von einem beliebigen Intervalle $\alpha \beta$ aus, so kann man immer ein im Innern von $\alpha \beta$ gelegenes Intervall $\alpha_{1} \beta_{1}$ bestimmen, so dass $f\left(\alpha_{1}\right)=f\left(\beta_{1}\right)$. Wenn die Function zwischen $\alpha_{1}$ und $\beta_{1}$ nicht constant ist - ein Fall, der leicht ausgeschlossen werden kann, -- dann nimmt sie jedenfalls Werte an, die größer oder kleiner sind als $f\left(\alpha_{1}\right)$. Wegen der Stetigkeit unserer Function gibt es nun in ihrem, dem Intervall $\alpha_{1} \beta_{1}$ entsprechenden Wertevorrath einen größten und einen kleinsten Wert, von denen wenigstens einer von $f\left(\alpha_{1}\right)$ verschieden ist, d. h. die Function besitzt in jedem (beliebig kleinen) Intervalle extreme (größte oder kleinste) Werte. Dies ist nur ein anderer Ausdruck für die früher festgesetzte Eigenschaft der Function $f(x)$. Denn wenn 
diese Function an der Stelle $\xi$ einen extremen Wert besitzt, und - wie vorausgesetzt - in der Umgebung von $\xi$ stetig bleibt, wird jeder, dem Werte $f(\xi)$ genügend nahe Wert, wenn er nur größer bezw. kleiner als $f(\xi)$ ist, an zwei in der Umgebung von $\xi$ liegenden Stellen, $\xi^{\prime}$ und $\xi^{\prime \prime}$, vorkommen, wo $\xi^{\prime}<\xi<\xi^{\prime \prime}$.

Diefolgenden Untersuchungensollen sichnun a uf einezwischen $A$ und $B$ gegebene und ebendort stetige Function $F(x)$ der reellen Variabeln $x$ beziehen, die in beliebiger Nähe einer beliebigen Stelle gleiche Werte annimmt, oder also auchwenn es kein endliches Intervall gibt, in dem die Function constant bleibt - in beliebiger Nähe einer beliebigen Stelle extreme Wertebesitzt.

2. Ist $F(x)$ eine Functionvon der betrachteten Beschaffenheit, so gibtes in jedem (noch sokleinen) Intervalle, das heibt in beliebiger Nähe einer beliebigen Stelle unendlich viele Stellen, an denen. die Function denselben Wert annimmt.

Das (selbstverständlich in $A B$ enthaltene) Intervall, von dem wir ausgehen, sei $a_{1} b_{1}$. Der Annahme nach gibt es in diesem. Intervalle $k$ Stellen, wo $k>1$,

$$
x_{1}, x_{2}, \ldots, x_{k}
$$

denen gleiche Functionalwerte entsprechen. Für jede dieser Stellen $x_{i}$ kann nun ein Intervall $x_{i}^{\prime} x_{i}^{\prime \prime}$ bestimmt werden, 'wo $\boldsymbol{x}_{i}^{\prime}<x_{i}<x_{i}^{\prime \prime}$, so dass die Intervalle

$$
x_{1}{ }^{\prime} x_{1}{ }^{\prime \prime}, x_{2}{ }^{\prime} x_{2}{ }^{\prime \prime}, \ldots, x_{k}{ }^{\prime} x_{k}{ }^{\prime \prime}
$$

keine gemeinsamen Stellen enthalten. Auch die Intervalle

$$
x_{1}{ }^{\prime} x_{1}, x_{1} x_{1}{ }^{\prime \prime}, x_{2}{ }^{\prime} x_{2}, x_{2} x_{2}{ }^{\prime \prime}, \ldots, x_{k}{ }^{\prime} x_{k}, x_{k} x_{k}{ }^{\prime \prime}
$$

werden daher mit Ausnahme der Grenzstellen $x_{1}, x_{2}, \ldots, x_{k}$ keine gemeinsame Stelle enthalten.

Die Function kann als in keinem noch so kleinem Theile dieser Intervalle constant vorausgesetzt werden, denn im entgegengesetzten Falle ist der zu beweisende Satz selbstverständlich richtig. Es gibt daher in jedem dieser Intervalle Funetionalwerte, die größer oder kleiner als $F\left(x_{1}\right)$ sind. Es sei die Anzahl der Intervalle, in denen Functionalwerte vorkommen, die größer, bezw. kleiner als $F\left(x_{1}\right)$ sind, $p$ und $q$. Jedes Intervall muss in eine dieser Klassen, kann aber auch in beide gehören; es ist daher

$$
p+q \geq 2 k
$$

und also wenigstens eine der beiden positiven ganzen Zahlen $p, q$ nicht kleiner als $k$.

Sei nun die Anzahl jener Intervalle, in denen Functionalwerte vorkommen, die $\underset{\substack{\text { gien er } \\ \text { kleiner }}}{\text { als }} F\left(x_{1}\right)$ sind, nicht kleiner als $k$, 
und diese Intervalle selbst in einfacherer Bezeichnung

$$
\alpha_{1} \beta_{1}, \alpha_{2} \beta_{2}, \ldots, \alpha_{r} \beta_{r},(r \geqq k) .
$$

An der einen Grenzstelle jedes Intervalls ist der Wert der Function gleich $F\left(x_{1}\right)$.

Nun gibt es in jedem dieser Intervalle eine Stelle, der

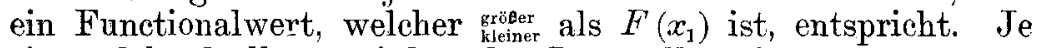
eine solche Stelle aus jedem der Intervalle sei

$$
y_{1}, y_{2}, \ldots, y_{r}
$$

Als stetige Function nimmt $F(x)$ im Intervall $\alpha_{1} \beta_{1}$ jeden zwischen $F\left(x_{1}\right)$ und $F\left(y_{1}\right)$ liegenden Wert an; man kann daher $y_{1}$ auch so wählen, dass $F\left(y_{1}\right) \underset{\text { króner }}{\text { klejer }}$ sei, als $F\left(y_{2}\right), \ldots, F\left(y_{r}\right)$. Beschränkt man sich auf eine genügend kleine Umgebung dieses $y_{1}$, so kann sogar ein Intervall $y_{1}{ }^{\prime} y_{1}{ }^{\prime \prime}$ angegeben werden $\left(a_{1}<y_{1}{ }^{\prime}<y_{1}<y_{1}{ }^{\prime \prime}<b_{1}\right)$, so dass an einer beliebigen Stelle dieses Intervalls der Wert der Function ${ }_{\text {greiner }}^{\text {kler }}$ als $F\left(y_{2}\right), \ldots$,

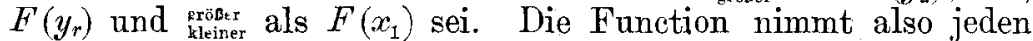
Wert, den sie im Intervall $y_{1}^{\prime} y_{1}^{\prime \prime}$ erhält, an wenigstens $r$ verschiedenen Stellen des Intervalls $a_{1} b_{1}$ an.

Da nun aber auch im Intervall $y_{1}^{\prime} y_{1}^{\prime \prime}$ die Function gleiche Werte annimmt, so kann man abermals zwei in diesem enthaltene Theilintervalle, $y^{\prime} y^{\prime \prime}$ und ein zweites $\alpha_{r+1} \beta_{r+1}$ bestimmen, so dass die Function jeden Wert, den sie im Intervalle $y^{\prime} y^{\prime \prime}$ annimmt, im ganzen an wenigstens $r+1(\geq k+1)$ verschiedenen Stellen des Intervalls $a_{1} b_{1}$ annimmt.

Da nun jeder Wert der Function aus dem Intervalle $y^{\prime} y^{\prime \prime}$ auch in den Intervallen $\alpha_{2} \beta_{2} \ldots \alpha_{r+1} \beta_{r+1}$ vorkommt, kann man dieses Verfahren wiederholen und wieder innerhalb $y^{\prime} y^{\prime \prime}$ ein Intervall $y^{(3)} y^{(4)}$ bestimmen, so dass jeder Stelle dieses Intervalls ein Functionalwert entspricht, der in $a_{1} b_{1}$ an wenigstens $r+2(\geq k+2)$ Stellen auftritt.

In dieser $W$ eise erhält man eine unbegrenzte Folge von Intervallen, deren jedes vollständig im Vorhergehenden enthalten ist:

$$
y^{\prime} y^{\prime \prime} ; y^{(3)} y^{(4)} ; \ldots ; y^{(2 n-1)} y^{(2 x)} \ldots \ldots \text {, }
$$

so dass jeder einer Stelle des Intervalls $y^{(2 n-1)} y^{(2 n)}$ entsprechende Functionalwert an wenigstens $k+n$ verschiedenen Stellen des Intervalls $a_{1} b_{1}$ auftritt. Dabei sind

$$
y^{\prime}, y^{(3)}, \ldots, y^{(2 n-1)} \text { und } y^{\prime \prime}, y^{(4)}, \ldots, y^{(2 n)}, \ldots
$$

solche Zahlenfolgen, wo immer

während noch

$$
y^{(2 n-1)} \leq y^{(2 n+1)} \text { und } y^{(2 n)} \geq y^{(2 n+2)},
$$

$$
y^{(2 x-1)}<y^{\prime \prime} \text { and } y^{\left(2 x^{\prime \prime}\right)}>y^{\prime} \text {. }
$$


Jede dieser Zahlenfolgen besitzt also einen Grenzwert, und setzt man endlich

$$
\lim y^{(2 n-1)}=z^{\prime}, \lim y^{(2 n)}=z^{\prime \prime},
$$

so enthalten alle Intervalle $y^{(2 n-1)} y^{(2 n)}$ das Intervall $z^{\prime} z^{\prime \prime}$ oder wenigstens die Stelle $z^{\prime}$, wenn $z^{\prime}=z^{\prime \prime}$. Jedenfalls existiert eine Stelle $z$, die in jedem Intervalle $y^{(2 n-1)} y^{(2 n)}$ enthalten ist. Dann gibt es aber unendlich viele Stellen im Intervalle $a_{1} b_{1}$, an denen die Function den Wert $F(z)$ besitzt.

Denn $z$ ist für jeden positiven ganzen Wert von $n$ im Intervall $y^{(2 n-1)} y^{(2 n)}$ enthalten, es gibt demnach wenigstens $k+n$ Stellen, wo die Function den Wert $F(z)$ annimmt. Nun wird $k+n$, wenn $n$ genügend groß gewählt wird, größer als jede beliebige Zahl $K$, und die Anzahl der Stellen, wo der Wert der Function gleich $F(z)$ wird, ist also größer als eine beliebige ganze Zahl $K$, d. h. unendlich groß.

3. Sei nun

$$
z_{1}, z_{2}, \ldots, z_{m}, \ldots
$$

eine unbegrenzte Folge von Stellen des Intervalls $a_{1} b_{1}$, an denen die Function $F(x)$ denselben Wert $F(z)$ annimmt; dann gibt es bekanntlich wenigstens eine Stelle $\xi$ von der Beschaffenheit, dass in beliebig kleiner Umgebung derselben immer noch unendlich viele Stellen $z_{m}$ existieren. Nan kann demnach eine unbegrenzte Folge von in $z_{1}, z_{2}, \ldots, z_{m}, \ldots$ enthaltenen Stellen

$$
\varsigma_{1}, \zeta_{2}, \ldots, \zeta_{n}, \ldots
$$

bestimmen, die für jedes $n$ von $\zeta$ verschieden sind, und für welche

$$
\lim . \zeta_{n}=\zeta
$$

nach dem Früheren ist noch

$$
F\left(\check{\zeta}_{n}\right)=F(z) \text {. }
$$

Da nun unsere Function an der Stelle $\zeta$ stetig ist, wird endlich auch

also

$$
F(\zeta)=\lim . F^{\prime}\left(\zeta_{n}\right)=F(z) ;
$$

oder wenn

gesetzt wird,

und

$$
\lim \frac{F(\zeta n)-F(\zeta)}{\zeta n-\zeta}=0,
$$

$$
\zeta_{n}=\zeta+h_{n}
$$

$$
\lim . h_{n}=0
$$

$$
\lim \cdot \frac{F\left(\zeta+h_{n}\right)-F(\zeta)}{h}=0 .
$$



fassen:

Man kann dieses Ergebnis in folgendem Satze zusammen-

Wenn die Function $F(x)$ zwischen den Grenzen $A$ und $B$ stetig ist, und in beliebig kleiner Umgebung einer beliebigen Stelle dieses Intervalls gleiche Werte annimmt, so kann man auch in beliebiger Nähe einer beliebigen Stelle solche $\zeta$ bestimmen, dass

$$
\lim \cdot \frac{F\left(\zeta+h_{n}\right)-F(\zeta)}{h_{n}}=0
$$

wird, wenn $h_{1}, h_{2}, \ldots, h_{n}, \ldots$ passend gewählte, der Grenze Null zustrebende Zablen sind.

4. Der soeben aufgestellte Satz beleuchtet in einfacher Weise das allgemeine Verhalten der merkwürdigen, von Weierstrass*) gegebenen Function

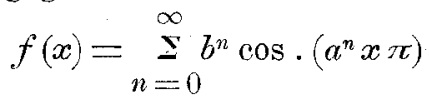

wo $a$ eine, von 1 verschiedene, ungerade ganze $Z a$ ahl, $b$ eine positive Constante und kleiner als 1 , und endlich noch $a$ und $b$ diesen Bedingungen gemäß so zu wählen sind, dass

wird.

$$
a b>1+\frac{3}{2} \pi
$$

Dann kann man, wie W eierstrass beweist, einer beliebigen Stelle $x_{0}$ entsprechend, zwei unbegrenzte Zahlenfolgen:

$$
x_{1}{ }^{\prime}, x_{2}{ }^{\prime}, \ldots, x_{m}{ }^{\prime}, \ldots \quad x_{1}{ }^{\prime \prime}, x_{2}{ }^{\prime \prime}, \ldots, x_{m}{ }^{\prime \prime}, \ldots
$$

bestimmen, für welche immer

und dabei auch

$$
x_{m}^{\prime}<x_{0}<x_{m}^{\prime \prime},
$$

$$
\lim . x_{m}{ }^{\prime}=\lim . x_{m}^{\prime \prime}=x_{0}
$$

wird, so dass bei Anwendung dieser Zahlen die beiden Differenzenquotienten

$$
\frac{f\left(x_{m}{ }^{\prime}\right)-f\left(x_{0}\right)}{x_{m}{ }^{\prime}-x_{0}} \text { und } \frac{f\left(x_{m}{ }^{\prime \prime}\right)-f\left(x_{0}\right)}{x_{m^{\prime \prime}}-x_{0}},
$$

wenn $m$ genügend groß gewählt wird, dem absoluten Betrage nach größer als irgendwelche beliebig gewählte Zahl werden und dabei immer verschiedenes Vorzeichen besitzen. Es kann demnach der Differentialquotient der Function $f(x)$ weder endlich und bestimmt, noch bestimmt (mit bestimmtem Vorzeichen) unendlich sein, d. h. die Function besitzt überhaupt an keiner Stelle einen Differentialquotienten.

*) S. D u Bois-Reymond's Mittheilung in Borchardt's Journal. Bd. 79, und auch Weierstrass, Abh. zur Functionentheorie. 
Die Anwendung der früher entwickelten Sätze ergibt nun noch Folgendes über das Verhalten dieser Function.

Dem Vorhergehenden nach sind $f\left(x_{m}^{\prime}\right)$ und $f\left(x_{m}{ }^{\prime \prime}\right)$ zugleich größer oder kleiner, als $f\left(x_{0}\right)$, und es gibt demnach in jedem noch so kleinen Intervalle Stellen, wo die Function gleiche Werte annimmt. Dieselbe Eigenschaft besitzt auch die allgemeinere Function

$$
\varphi(x)=f(x)-C x,
$$

wo $C$ eine beliebig gewählte Constante bedeutet, wie sich unmittelbar aus

ergibt.

$$
\frac{p\left(x_{m}\right)-\varphi\left(x_{0}\right)}{x_{m}-x_{0}}=\frac{f\left(x_{m}\right)-f\left(x_{0}\right)}{x_{m}-x_{0}}-C
$$

Es gibt demnach in beliebiger Nähe der beliebigen Stelle $x_{0}$ auch Stellen $\xi$, für welche

$$
\frac{\varphi\left(\xi+h_{n}\right)-\varphi(\xi)}{h_{n}}=\frac{f\left(\xi+h_{n}\right)-f(\xi)}{h_{n}}-C
$$

Null wird, wenn die Zahlen $h_{1}, h_{2}, \ldots, h_{n}, \ldots$ in passender Weise der Grenze 0 zustreben, oder endlich:

In beliebiger Nähe derwillkürlich gewählten Stelle $x_{0}$ gibt es auch Stellen $\xi$, für welche der Differenzenquotient

$$
\frac{f\left(\xi+h_{n}\right)-f(\xi)}{h_{n}}
$$

sich der willkürlich gewählten bestimmten Zahl $C$ nähert, wenn die Incremente $h_{n}$ sich in gewisser bestimmter Weise der Grenze Null nähern.

Bei anderer Wahl der $h_{n}$ wird für dasselbe $\xi$ jener Quotient positiv oder negativ unendlich, und es ist also ohne weiteres rechnerisches Verfahren gezeigt, dass die Grenze des Differenzenquotienten für unsere Function an beliebig vielen Stellen eines beliebig kleinen Intervalls völlig unbestimmt ist, und innerhalb dieses Intervalls, wenn nur der Grenzïbergang in entsprechender Weise ausgeführt wird, jeden beliebigen Wert aunehmen kann.

Man kann ibrigens auf Grundlage der hier angestellten Betrachtungen auch den strengen Beweis dafür liefern, dass stetige Functionen, deren Differentialquotient an jeder Stelle eines gegebenen Intervalls (bestimmt oderunbestimmt) unendlieh wird, iiberhaupt nicht existieren. 